\title{
Do Femoral Neck and Stem Anteversion Affect Final Femur Rotation and Pelvic Positioning after Total Hip Arthroplasty?
}

\author{
Norio Imai", Tomoyuki Ito, Yasuhito Takahashi, Yoji Horigome, Ken Suda, Dai Miyasaka, \\ Naoto Endo \\ Department of Orthopedic Surgery, Niigata University Medical and Dental Hospital, Niigata, Japan. \\ Email: *imainorio2001@yahoo.co.jp
}

Received May $13^{\text {th }}, 2013$; revised June $14^{\text {th }}, 2013$; accepted June $26^{\text {th }}, 2013$

Copyright (C) 2013 Norio Imai et al. This is an open access article distributed under the Creative Commons Attribution License, which permits unrestricted use, distribution, and reproduction in any medium, provided the original work is properly cited.

\begin{abstract}
In this study, we have investigated the rotation of the pre-operative and post-operative femur, and stem anteversion to prevent impingement and dislocation. We studied 126 patients who had undergone primary total hip arthroplasty (THA) for the treatment of osteoarthritis. We compared preoperative tilt of anterior pelvic plane (APP), posterior condylar axis of the femur (PCA) relative to APP (APP-PCA) with those of postoperative ones in supine position. There was a moderate correlation between the pre- and post-operative APP-PCA values $(r=0.71)$. The postoperative APP-PCA decreased by approximately $6^{\circ}$. However, there was poor correlation between the stem anteversion and the postoperative APP-PCA $(r=-0.27)$. A greater reduction in APP-PCA was observed among patients who exhibited greater external rotation of femur compared to those who showed less external rotation in supine position before THA. We also observed that the post-operative rotation of the femur was dependent on the pre-operative rotation of the femur while it was independent of the stem anteversion.
\end{abstract}

Keywords: Anterior Pelvic Plane; Posterior Condylar Axis; Rotation; Total Hip Arthroplasty

\section{Introduction}

The orientation of the acetabular and femoral components in total hip arthroplasty (THA) is related to satisfactory postoperative outcomes [1,2]. Malpositioning in THA may cause impingement between the acetabular and femoral components, with subsequent dislocation, accelerated wearing away of polyethylene and hip pain [2].

Various techniques are being developed to optimize the orientation of the acetabular component during THA. In recent years, combined anteversion, which is anteversion of the acetabular and femoral components, has been proposed $[3,4]$, rather than alignment of the acetabular component only [5]. However, the positions of the pelvis and femur are dynamic during standing, lying, sitting, and other daily activities, and during the pre- and postoperative periods, it has been demonstrated that pelvic flexion and rotation of the femur substantially affects the orientation of the acetabular cup and femoral stem $[6,7]$.

"Corresponding author.
Therefore, we believe that the angle of both the acetabular and femoral components should be selected on an individual basis and also the implantation surgeons are required to make precise adjustments to achieve the optimal degrees of pelvic tilt and rotation of the femur.

There is substantial evidence on the effect of pelvic flexion on the cup anteversion demonstrated on anteroposterior or lateral radiography or computed tomography (CT) [6-8], but the effect of femur rotation on stem anteversion has not been established. We selected patients with osteoarthritis of the hip to compare pre- and postoperative variation in the rotation of the femur and pelvic flexion. We chose to observe the patient in the supine position because this approach offers higher stability of the implants and lower risks of hip dislocation. Many surgeons insert the stem at the same anteversion to all the patients, while the patient has a different rotation of the femur. For example, if the femur was more externally rotated, posterior impingement and anterior dislocation is more likely to occur. Therefore, in this study we have investigated the rotation of the pre-operative and post-op- 
erative femur, and stem anteversion to prevent impingement and dislocation. Moreover, we also investigated whether there was a difference between the pre- and post-operative change in the tilt of the pelvis and rotation of the femur and whether there was a correlation between stem anteversion and post-operative rotation of the femur.

\section{Materials and Methods}

\subsection{Subjects}

We studied 126 patients attending our institution, who had undergone primary THA for the treatment of osteoarthritis of the hip joint between February 2009 and August 2011. The ethics committee of Niigata University Medical and Dental Hospital approved the present study and an informed consent was obtained from each patient. Patients were excluded from the study if they had previously undergone hip surgery or they were suffering from osteonecrosis or rheumatoid arthritis, knee osteoarthritis with flexion contracture, or if they had undergone adductor tenotomy to restrict abduction. We included patients with bilateral coxoarthritis after they had undergone the second THA while the pelvic tilt and rotation of the femur of first THA was not included in this study. Our final study sample comprised 28 men and 98 women. The average age of the patients was $58.7 \pm 9.3$ (range: 40 75 years). There were 99 patients classified as Crowe [9] grade I, 23 as grade II and 4 as grade III dysplasia.

\subsection{Zed Hip System}

We used the software Zed Hip ${ }^{\circledR}$ (Lexi, Tokyo) to create a 3D digital bone model that accurately reproduced the spatial relationship between the pelvis and the femur, and to calculate the various alignment parameters within $1^{\circ}$ and $1 \mathrm{~mm}$ of accuracy [10]. We used multislice CT with $1.25 \mathrm{~mm}$ intervals to examine the area from the most proximal part of the pelvis to the most distal part of the femur. The patient was imaged in the supine position where the knees were fully extended, feet in a comfortable position and hands on the stomach. Several bony landmarks were then digitized to establish reference points and anatomic coordinate systems. For the pelvis, the anterior pelvic plane (APP) was obtained by digitizing the anterior superior iliac spine (ASIS) on each side and the pubic symphysis. The origin of the pelvic anatomic coordinate system was defined as the pubic symphysis. The pelvic $\mathrm{X}$ axis $\left(\mathrm{X}_{\mathrm{p}}\right.$ axis) was the transverse axis, defined as a parallel line from the left ASIS to the right ASIS. The $Z_{p}$ axis was perpendicular to the $X$ axis in the plane of the APP. The $Y_{p}$ axis was perpendicular to the APP.

A similar procedure was used to digitize reference points for the femoral coordinate system. The femoral $\mathrm{X}$ axis $\left(\mathrm{X}_{\mathrm{f}}\right.$ axis) was defined as the line connecting the most posterior points of the medial and lateral posterior femoral condyles. The origin of the femoral coordinate system was defined as the midpoint of the line containing the most posterior points of the bilateral posterior condyles. The $Z_{f}$ axis was perpendicular to the $X_{f}$ axis and in a table top plane, which consisted of the most posterior points of the bilateral posterior condyles and the most prominent posterior point of the greater trochanter. The $Y_{f}$ axis was defined as the cross section of the $Z_{f}$ axis and the $\mathrm{X}_{\mathrm{f}}$ axis.

\subsection{Implantation}

Based on prior reports [4], we planned to place both components as follows. The acetabular component was placed at approximately $10^{\circ}-20^{\circ}$ of anteversion and $40^{\circ}$ inclination, relative to the APP according to the radiographic definition of Murray [7]. The anteversion of the acetabular component was compensated depending on the angle of pelvic flexion. The femoral component was placed at approximately $15^{\circ}-25^{\circ}$ of anteversion relative to the posterior condylar axis (PCA), aiming at $35^{\circ}-40^{\circ}$ of combined anteversion.

The THA operations were performed by experienced surgeons (NI, TI, KS and DM) according to a standard protocol using the Watson-Jones anterolateral approach in with the patient lying completely supine. For all patients, we used a single uncemented acetabular and femoral component (BiCONTACT Hip System, AES-CLUP, Tuttlingen, Germany), and all patients followed the same post-operative rehabilitation protocol. All the patients were permitted to leave the bed from second post-operative day. Subsequently they were permitted full weight bearing if they could.

\subsection{CT Examination}

Multislice CT was performed at all of the participants preoperatively to locate the position of both acetabular and femoral components with Zed Hip system and one week after THA to confirm the position of acetabular and femoral components with Zed Hip system. Thirty-two out of the 126 patients in our study sample had repeat CT at 12 weeks or later (average: 16, range: 12 - 66 weeks) for the purpose of follow up. We investigated the differences between the position of the pelvis and femur at 1 and 12 weeks after THA in order to verify the effect of the postoperative period with 32 joints currently examined twice or more.

\subsection{Measurement}

We measured the anteversion and inclination of aceta- 
bular cup according to the Murray's radiographic definition [8]. We defined the femoral neck axis according to the method described by Sugano et al. [11]. We identified 3 circles on the femoral neck just below the femoral head, tangential to the anterior and posterior margins of the femoral neck. The definition of the three circles was as follows: first, a circle was described close to the junction of the femoral neck and shaft. Next, a line between the center of this circle and the proximal end of the femoral neck was divided approximately into 3 , and 2 further circles were defined at the approximate $1 / 3$ and $2 / 3$ points respectively. The femoral neck axis was de- fined by the line connecting the centers of these 3 circles (Figure 1). Femoral neck anteversion (FNA) was defined as the angle connecting the femoral neck axis and the PCA (Figure 2).

Stem anteversion (SA) was defined as the angle connecting the stem axis and the PCA, in the table top plane, similar to the measurement of FNA. Lastly, we measured PCA relative to APP (APP-PCA), as the angle that connected the PCA projected on the XYp plane and the $\mathrm{Xp}$-axis (Figure 3). All the measurement was performed twice by one observer and the average value was adopted.

\subsection{Statistical Analysis}

To assess intraobserver variation, we calculated the average of 2 repeat image measurements performed by one of the authors (NI). We compared the measurements made by NI and YT in a subset of 25 patients randomly selected from our study population to assess the interobserver variation. We analyzed the data using SPSS statistical software (SPSS, Inc, Chicago, IL). Differences between pre- and postoperative values were analyzed using the paired $t$ test and we analyzed correlation using Pearson's coefficients. We used the Bonferroni correction ( $\alpha$ $=2$ ) to adjust the pre- and postoperative differences in the tilt of APP ( $\triangle$ APP) and APP-PCA ( $\triangle$ APP-PCA). For the analysis of intra- and interobserver variation, we calculated the interclass correlation coefficients (ICC). A $\mathrm{p}$ value $<0.05$ was considered statistically significant.

\section{Results}

Table 1 shows the mean values of measurement of the hip.

The anteversion and inclination of acetabular cup was similar in both sexes.

The tilt of APP after THA $\left(2.6^{\circ} \pm 5.1^{\circ}\right)$ was significantly smaller than the preoperative tilt of APP $(5.4 \pm$ $\left.7.3^{\circ}\right)$ and there was strong correlation between the measurements $(r=0.92)$ (Figure 4). The postoperative tilt of APP decreased by approximately $3^{\circ}$ and the formula of the regression line was as follows:

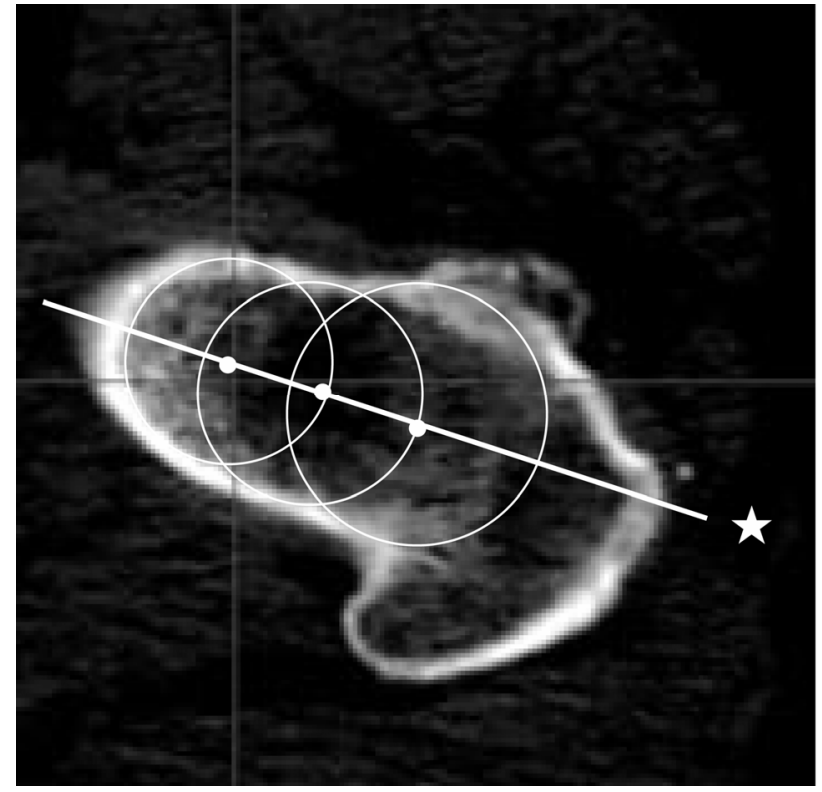

Figure 1. Definition of the femoral neck axis. The femoral neck axis $(\star)$ was defined as the line connecting the centers of the 3 circles along the femoral neck, below the femoral head, and tangential to the anterior and posterior margins of the femoral neck.

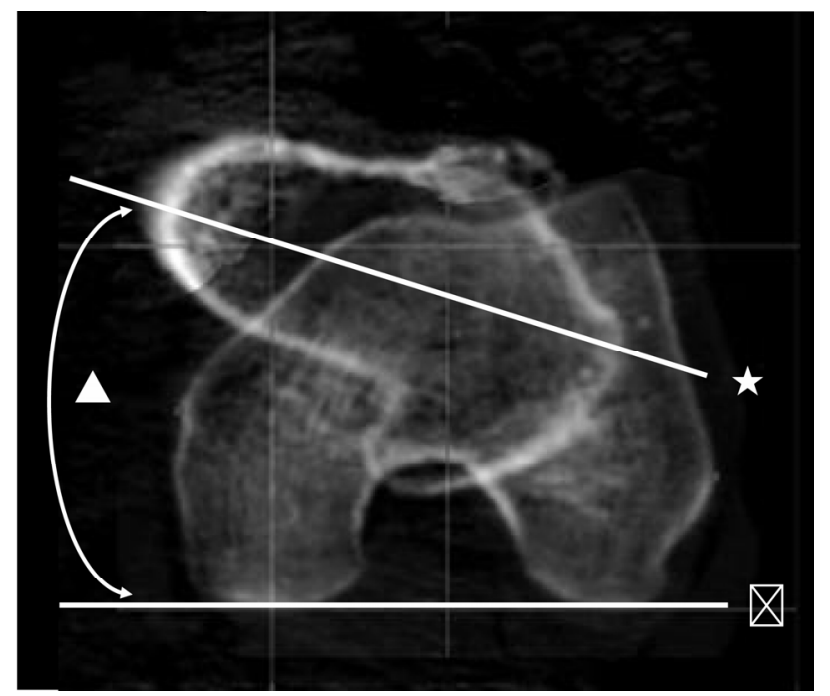

Figure 2. Measurement of femoral neck anteversion (FNA). The FNA $(\Delta)$ is the angle between the femoral neck axis $(\star)$ and the posterior condylar axis (\#).

$($ postoperative tilt of APP $)=0.68 \times($ preoperative tilt of APP) $-0.98^{\circ}$

There were significant pre- and post-operative differences in $\triangle$ APP in both men and women, but the magnitude of change was similar in both sexes (Table 1).

Next, the variation of APP-PCA, PCA relative to APP, was measured, which gave the rotation of the femur relative to the pelvis. The pre and postoperative APP-PCA 


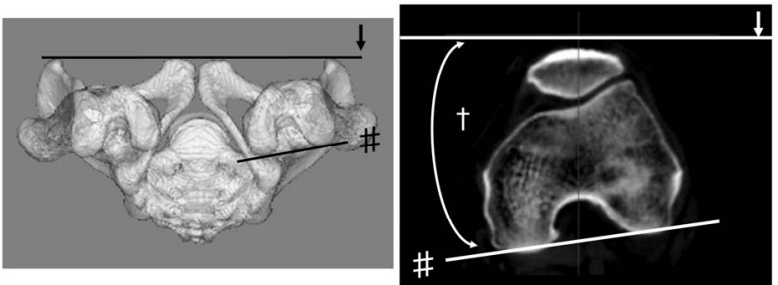

Figure 3. Measurement of the anterior pelvic plane-posterior condylar axis (APP-PCA) The APP-PCA ( $\dagger$ ) was defined as the angle connecting the APP $(\downarrow)$ and the PCA projected on the XY plane of the APP (\#).

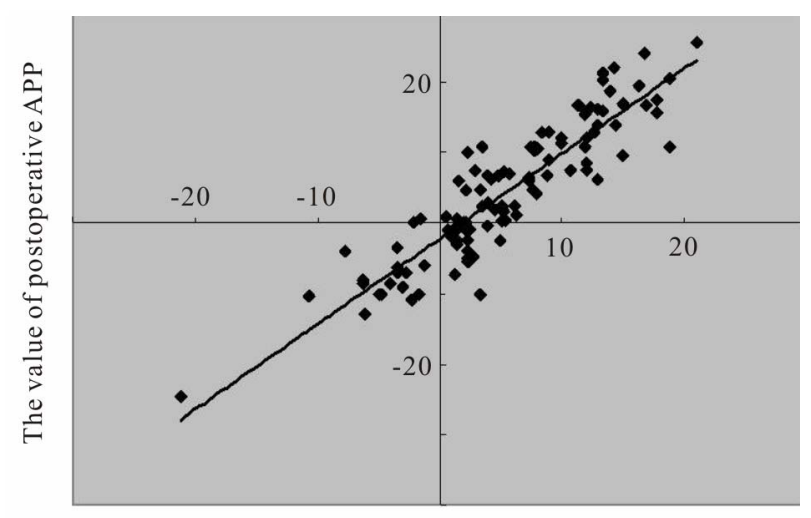

The value of preoperative APP

Figure 4. Correlation between measurements of the anterior pelvic plane (APP) performed before and after total hip arthroplasty (THA).

was $11.0^{\circ} \pm 11.4^{\circ}$ and $4.8^{\circ} \pm 9.2^{\circ}$ respectively. The change was significant $(\mathrm{p}<0.001)$ and there was moderate correlation between the values $(\mathrm{r}=0.71)$ (Figure 5). The postoperative APP-PCA decreased by approximately $6^{\circ}$ and the formula of the regression line was as follows:

$($ Postoperative APP-PCA $)=0.57 \times($ Preoperative APP-PCA) $-5.40^{\circ}$

There was a trend towards greater reduction in APPPCA among patients with greater external rotation before THA compared to those with less external rotation.

There were significant pre- and post-operative differences in $\triangle$ APP-PCA in both men and women, but the magnitude of change was similar in both sexes (Table 1). There was poor correlation between the SA and the postoperative APP-PCA $(r=-0.27)$ (Figure 6). In other words, it meant that the variation of postoperative rotation of the femur was independent of the anteversion of femoral component.

Moreover, there was a poor correlation between the difference of FNA and SA and $\triangle$ APP-PCA $(\mathrm{r}=-0.16)$ (Figure 7). In other words, it meant that the variation of pre- to post-operative rotation of the femur was independent of the variation of anteversion of femur to the anteversion of femoral component.

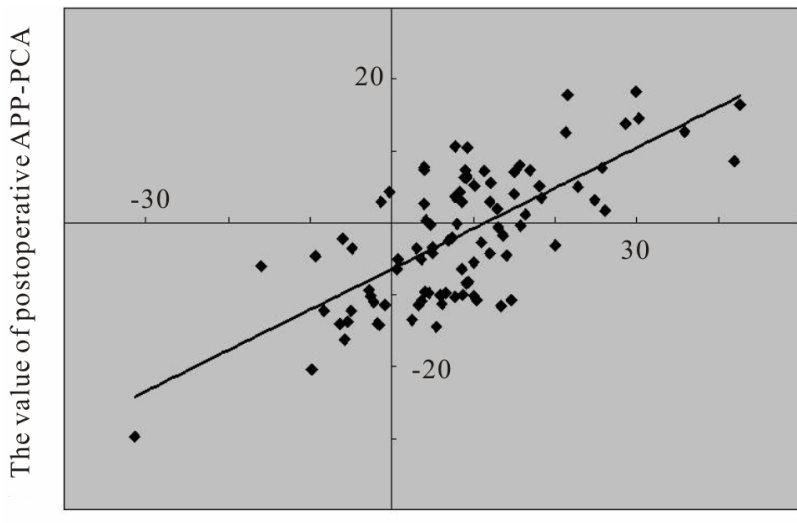

The value of preoperative APP-PCA

Figure 5. Correlation between measurements of the anterior pelvic plane posterior condylar axis (APP-PCA) performed before and after total hip arthroplasty (THA).

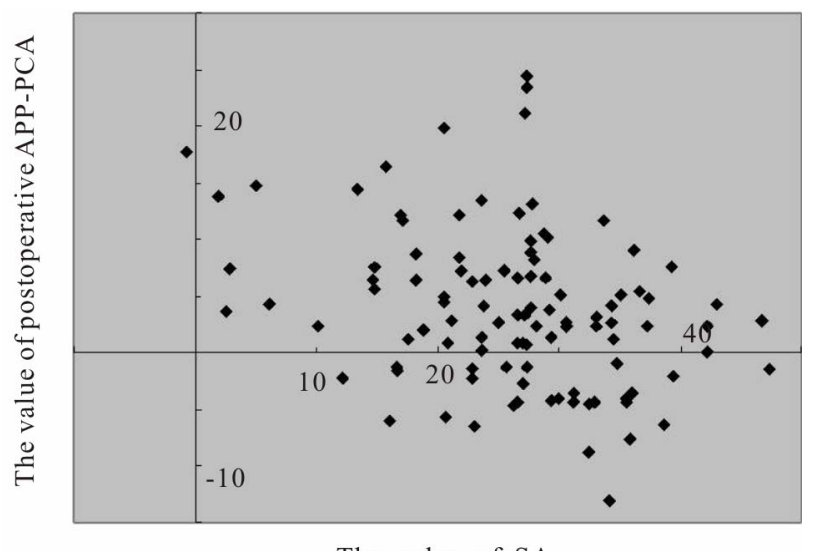

The value of SA

Figure 6. Correlation between measurements of stem anteversion (SA) and the anterior pelvic plane-posterior condylar axis (APP-PCA) performed after total hip arthroplasty (THA).

The absolute values for the tilt of APP among the 32 patients who underwent repeat CT after 12 weeks or more, did not differ significantly at the second measurement $\left(2.1^{\circ} \pm 1.3^{\circ}, 0.5-4.0\right)$ to those obtained 1 week after THA $\left(3.1^{\circ} \pm 3.6^{\circ}\right)$ The difference between the first and the second measurements of the tilt of APP (p = $0.408, r=0.964)$ or APP-PCA $(p=0.832, r=0.981)$ was not statistically significant. None of the participants in this study, showed any dislocation.

According to these results, if the surgeons aim to insert the femoral component at $20^{\circ}$ of anteversion relative to the PCA and acetabular component at $20^{\circ}$ of anteversion relative to post-operative tilt of APP, combined anteversion was $40^{\circ}$, the implantation angles of acetabular and femoral component was as follows;

1) anteversion of acetabular component $=20.98+0.68$ $\times$ (preoperative tilt of APP). 
Table 1. Mean values for measurements of the hip.

\begin{tabular}{cccc}
\hline & All patients $(\mathrm{n}=126)$ & Men $(\mathrm{n}=29)$ & Women $(\mathrm{n}=97)$ \\
\hline Anteversion of acetabular cup & $21.4 \pm 6.1(3.0,47.6)$ & $19.6 \pm 4.4(10.7,34.8)$ & $22.3 \pm 7.2(3.0,47.6)$ \\
Inclination of acetabular cup & $44.6 \pm 6.1(32.0,58.3)$ & $44.3 \pm 3.3(38.2,53.7)$ & $44.8 \pm 6.4(32.0,58.3)$ \\
FNA & $27.5 \pm 12.4(-15.6,77.2)$ & $22.5 \pm 12.5(-15.6,46.9)$ & $29.6 \pm 14.6(-5.5,77.2)$ \\
SA & $25.4 \pm 9.5(-0.7,52.9)$ & $24.1 \pm 11.1(-0.7,46.7)$ & $26.2 \pm 10.6(1.9,52.9)$ \\
Tilt of APP (before THA) & $5.4 \pm 7.3(-21.2,20.9)$ & $3.2 \pm 4.7(-6.2,12.2)^{*}$ & $6.3 \pm 7.6(-21.2,20.9)^{*}$ \\
Tilt of APP (after THA) & $2.6 \pm 5.1(-19.0,17.3)$ & $1.0 \pm 4.3(-5.2,11.4)^{*}$ & $3.5 \pm 6.4(-19.0,17.3)^{*}$ \\
$\Delta$ APP & $2.5 \pm 2.9(-7.6,5.6)$ & $2.2 \pm 1.8(-3.2,3.4)$ & $2.8 \pm 3.1(-7.6,5.6)$ \\
APP-PCA (before THA) & $11.0 \pm 11.4(-33.5,42.6)$ & $13.3 \pm 10.4(-15.9,42.6)^{*}$ & $9.2 \pm 13.5(-33.5,30.2)^{*}$ \\
APP-PCA (after THA) & $4.8 \pm 9.2(-35.6,20.2)$ & $7.4 \pm 8.3(-11.3,20.2)^{*}$ & $3.4 \pm 10.4(-35.6,18.2)^{*}$ \\
$\Delta$ APP-PCA & $6.2 \pm 9.0(-23.3,9.9)$ & $5.9 \pm 7.6(-18,9.9)$ & $5.8 \pm 9.3(-23.3,7.1)$ \\
\hline
\end{tabular}

Results are presented as mean \pm SD (range); ${ }^{*}$ p $<0.05$; FNA: femoral neck anteversion, SA: stem anteversion, APP: anterior pelvic plane, $\Delta$ APP: difference in the tilt of APP before and after total hip arthroplasty (THA); PCA: posterior condylar axis; APP-PCA: the angle that connecting the PCA projected on the XY plane and the pelvic $\mathrm{X}$-axis, $\triangle$ APP-PCA: the difference in APP-PCA before and after THA.

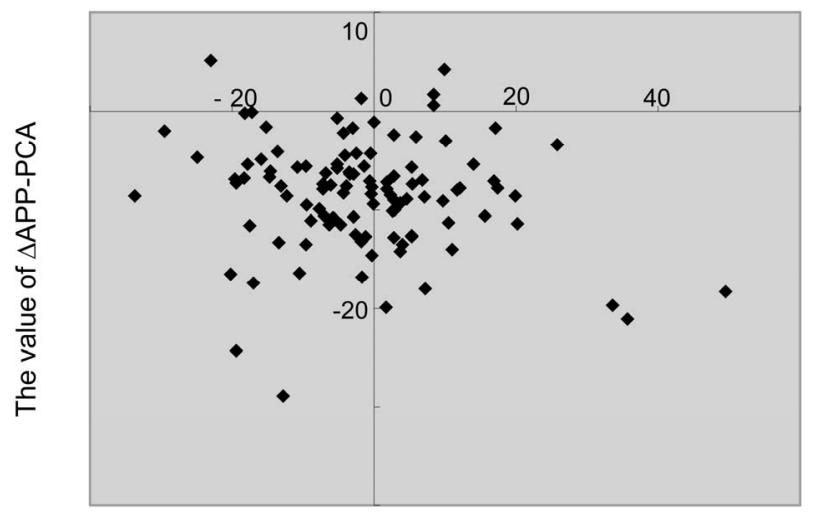

The value of difference of FNA and SA

Figure 7. Correlation between measurements of difference between FNA (femoral neck anteversion) and SA (stem anteversion) and pre- and postoperative differences in the anterior pelvic plane-posterior condylar axis ( $\triangle \mathrm{APP}-\mathrm{PCA})$.

2) anteversion of femoral component $=14.6-0.57 \times$ (Preoperative APP-PCA).

The maximum intra- and interobserver differences were observed in the measurement of FNA. The maximum intraobserver difference was $3.3^{\circ}$, and the largest standard deviation was $1.68^{\circ}$ (ICC: 0.959). The maximum interobserver difference was $3.4^{\circ}$, and the largest standard deviation was $1.73^{\circ}(\mathrm{r}=0.996, \mathrm{p}<0.001)$.

\section{Discussion}

Evidence suggests that combined anteversion of the acetabular and femoral components are important to avoid impingement of the two components [4]. However, these computer simulations do not take into account the inherent variability in bone orientations between patients, which may lead to implant instability and hip dislocation. Currently, there are no reports, however, investigating the rotation of the pre-operative and post-operative femur, and stem anteversion. In the present study, we conducted 126 successful THA using a procedure that takes into account the natural orientation of each patient's pelvis and femur with the Watson-Jones anterolateral approach.

During the THA, the orientation of the pelvic bone dictates the correct positioning of the acetabular implant. Therefore, minor variations in the pelvic bone orientation of a patient may contribute to implant instability. The present study shows that pelvic flexion varied by $5.4^{\circ}$ before THA, and $2.6^{\circ}$ post-operation. This variability is consistent with the $-4^{\circ}$ to $+5^{\circ}$ range previously reported in the supine position $[12,13]$.This indicates that pelvic flexion is not usually parallel to the coronal plane when the patient is in the supine position. Wan et al observed that only $9 \%$ of a study population of 619 patients did not have anteroposterior tilt when lying in the supine position [14], which is also in keeping with our results.

With regard to the postoperataive rotation of the femur, we demonstrated that the femur was externally rotated at approximately $11^{\circ}$ pre-operatively and approximately $5^{\circ}$ internally rotated compared to the pre-operative measurement. Surgeons who use the 3D navigation system may frequently place the femoral component relative to the PCA as well to bony landmarks [4]. We have demonstrated that the rotation of the femur is unique to different individuals, thus anteversion should be specifically selected for each patient.

The magnitude of change of the tilt of pelvis and the rotation of the femur did not depend on gender, but depended on the preoperative rotation of the femur. Moreover, postoperative rotation of the femur was independent of the stem anteversion and the difference of FNA and SA was also independent of $\triangle$ APP-PCA. Hence, the rotation may be related to the tension of the soft tissue 
around the hip joint, for example the iliopsoas muscle, tensor fascia lata, gluteus medius adductors, and so on, or to the length of the neck of the stem.

There are some limitations to our study. First, the CT examinations were performed one week after THA. However, we demonstrated that there were no significant differences between measurements made at the first and second examinations, and thus, we consider our data valid. We have only provided evidence for measurements made with the patient in the supine position, and further investigations are required to assess the variation in measurements obtained in other positions such as standing and sitting. Second, we performed all THAs using the Watson-Jones anterolateral approach. The surgeons approached intramuscularly to the hip joint without transection or detachment of muscles such as gluteus medius and short rotators in this method. This approach may affect the postoperative rotation. Third, in this study, we did not examine the relationship between the rotation of the femur and the change of limb length before and after THA. If we strictly examine the leg length elongation 3-dimensionally, it will be necessary to reconcile both the tilt of the pelvis and the rotation of the femur before THA with that after THA and compare the elongation in anteroposterior, mediolateral, and craniocaudal direction. It is not easy to compare them. Therefore, further examination will be required.

In conclusion, we recommend that the placement of the acetabular and femoral components is determined on an individual patient basis, by taking into account both the angle of pelvic flexion and the rotation of the femur, even if the optimal angle is calculated using mathematical formula [4]. We believe this approach will be able to estimate post-operative rotation of the femur and calculate the optimal stem anteversion, subsequently produce a larger range of motion at the hip and reduce the incidence of impingement and dislocation after THA.

\section{REFERENCES}

[1] F. J. Kummer, S. Shah, S. Iver and P. E. DiCesare, "The Effect of Acetabular Cup Orientations on Limiting Hip Rotation," The Journal of Arthroplasty, Vol. 14, No. 4, 1999, pp. 509-513. doi:10.1016/S0883-5403(99)90110-9

[2] A. Malik, A. Maheshwari and L. D. Dorr, "Impingement with Total Hip Replacement," The Journal of Bone \& Joint Surgery (American), Vol. 89, No. 8, 2007, pp. 18321842. doi:10.2106/JBJS.F.01313

[3] L. D. Dorr, A. Malik, M. Dastane and Z. Wan, "Combined Anteversion Technique for Total Hip Arthroplasty," Clinical Orthopaedics and Related Research, Vol. 467, No. 1, 2009, pp. 119-127. doi:10.1007/s11999-008-0598-4

[4] K. H. Widmer and B. Zurfluh, "Compliant Positioning of
Total Hip Components for Optimal Range of Motion," Journal of Orthopaedic Research, Vol. 22, No. 4, 2004, pp. 815-821. doi:10.1016/j.orthres.2003.11.001

[5] G. E. Lewinnek, J. L. Lewis, R. Tarr, C. L. Compere and J. R. Zimmerman, "Dislocations after Total Hip-Replacement Arthroplasties," The Journal of Bone \& Joint Surgery (American), Vol. 60, No. 2, 1978, pp. 217-220.

[6] A. M. DiGioia, M. A. Hafez, B. Jaramaz, T. J. Levison and J. E. Moody, "Functional Pelvic Orientation Measured from Lateral Standing and Sitting Radiographs," Clinical Orthopaedics and Related Research, Vol. 453, 2006, pp. 272-276. doi:10.1097/01.blo.0000238862.92356.45

[7] S. Parratte and J. N. Argenson, "Validation and Usefulness of a Computer-Assisted Cup-Positioning System in Total Hip Arthroplasty. A Prospective, Randomized, Controlled Study," The Journal of Bone \& Joint Surgery (American), Vol. 89, No. 3, 2007, pp. 494-499. doi:10.2106/JBJS.F.00529

[8] D. W. Murray, "The Definition and Measurement of Acetabular Orientation," The Journal of Bone \& Joint Surgery (British), Vol. 75, No. 2, 1993, pp. 228-232.

[9] J. F. Crowe, V. J. Mani and C. S. Ranawat, "Total Hip Replacement in Congenital Dislocation and Dysplasia of the Hip," The Journal of Bone \& Joint Surgery, Vol. 61, No. 1, 1979, pp. 15-23.

[10] K. Kobayashi, M. Sakamoto, Y. Tanabe, A. Ariumi, T. Sato, G. Omori and Y. Koga, "Automated Image Registration for Assessing Three-Dimensional Alignment of Entire Lower Extremity and Implant Position Using BiPlane Radiography," Journal of Biomechanics, Vol. 42, No. 16, 2009, pp. 2818-2822. doi:10.1016/j.jbiomech.2009.08.022

[11] N. Sugano, P. C. Noble, E. Kamaric, J. K. Salama, T. Ochi and H. S. Tullos, "The Morphology of the Femur in Developmental Dysplasia of the Hip," The Journal of Bone \& Joint Surgery (British), Vol. 80, No. 4, 1988, pp. 711-719. doi:10.1302/0301-620X.80B4.8319

[12] S. Blendea, K. Eckman, B. Jaramaz, T. J. Levison and A. M. Digioia, "Measurements of Acetabular Cup Position and Pelvic Spatial Orientation after Total Hip Arthroplasty Using Computed Tomography/Radiography Matching," Computer Aided Surgery, Vol. 10, No. 1, 2005, pp. 37-43.

[13] S. Nishihara, N. Sugano, T. Nishii, K. Ohzono and H. Yoshikawa, "Measurements of Pelvic Flexion Angle Using Three-Dimensional Computed Tomography," Clinical Orthopaedics and Related Research, 2003, Vol. 411, pp. 140-151. doi:10.1097/01.blo.0000069891.31220.fd

[14] Z. Wan, A. Malik, B. Jaramaz, L. Chao and L. D. Dorr, "Imaging and Navigation Measurement of Acetabular Component Position in THA," Clinical Orthopaedics and Related Research, Vol. 467, No. 1, 2009, pp. 32-42. doi:10.1007/s11999-008-0597-5 